PROCEEDINGS OF THE

AMERICAN MATHEMATICAL SOCIETY

Volume 133, Number 5, Pages 1469-1473

S 0002-9939(04)07670-1

Article electronically published on December 6, 2004

\title{
INTEGRABLE FACTORS IN COMPACT SCHUR MULTIPLIERS
}

\author{
MATS ERIK ANDERSSON
}

(Communicated by Joseph A. Ball)

\begin{abstract}
It is shown that a Schur multiplier is compact if and only if it is the Schur product of two multipliers, one of which is a Hankel-Schur multiplier generated by an integrable function. This is illuminated by factoring exotic, singular measures and is brought into relation with Paley set-based multipliers.
\end{abstract}

As a model of general, separable Hilbert spaces the classical space $\ell^{2}=\ell^{2}(\mathbb{N})$ is universal and operators on $\ell^{2}$ are conveniently identified with infinite matrices as determined by the action on the standard coordinate basis $\left\{e_{n}\right\}_{n=0}^{\infty}$. Matrices will be indexed by non-negative integers in this essay. A matrix $A=\left\{a_{j k}\right\}$ is a Schur multiplier if the Schur (or entrywise) product $B \mapsto A * B=\left\{a_{j k} b_{j k}\right\}$ maps the full space of bounded operators $B\left(\ell^{2}\right)$ into itself. The collection of all Schur multipliers here denoted $V_{2}$ - becomes a commutative Banach algebra when given the operator norm as acting on $B\left(\ell^{2}\right)$ and supplied with the usual addition and the Schur product as multiplication. The multiplier norm will be denoted \|\|$_{m}$. Bennett $[\mathrm{Be}$ is an excellent reference on Schur multipliers. Some additional considerations - see Hladnik [H] for a concise presentation - reveals that $V_{2}$ coincides with the multiplier space of itself (acting via the Schur product) and this with identical norms. Thus the functional analytic properties of $A: B\left(\ell^{2}\right) \rightarrow B\left(\ell^{2}\right)$ and $A: V_{2} \rightarrow V_{2}$ coincide.

In order to simplify later formulations the notation $\Gamma_{\phi}$ for the Hankel matrix $\{\phi(j+k)\}_{j, k \geq 0}$ determined by the diagonal $\phi \in \ell^{\infty}(\mathbb{N})$ will be used. The subspace of $V_{2}$ consisting of Hankel matrices is clearly a closed subalgebra of $V_{2}$. The corresponding diagonals induce a Banach algebra $M^{H}$ of sequences in $\ell^{\infty}$ with norm $\|c\|_{M^{H}}=\left\|\Gamma_{c}\right\|_{m}$. It can be demonstrated by various means that

$$
\|c\|_{\ell^{\infty}} \leq\|c\|_{M\left(H^{1}\right)} \leq\|c\|_{M^{H}} \leq\|c\|_{B(\mathbb{N})}
$$

holds. Here $M\left(H^{1}\right)$ is the coefficient multiplier space of the Hardy space $H^{1}$, and $B(\mathbb{N})$ is the restricted Fourier-Stieltjes algebra with norm

$$
\|c\|_{B(\mathbb{N})}=\inf \left\{\|\rho\|_{M(\mathbb{T})} ;\left.\hat{\rho}\right|_{\mathbb{N}}=\left.c\right|_{\mathbb{N}}\right\}
$$

The purpose of this paper is to recognize the compact Schur multipliers in surprisingly sturdy terms. It was proved in $[\mathrm{H}]$ that the subalgebra of compact Schur multipliers can be characterised as the tensor product $c_{0} \otimes^{h} c_{0}$ supplied with the Haagerup tensor norm. A completely different characterisation will be achieved below.

Received by the editors October 8, 2003 and, in revised form, February 1, 2004.

2000 Mathematics Subject Classification. Primary 47A68; Secondary 47B07, 42A38, 43 A46.

Key words and phrases. Riesz products, Paley sets, Littlewood multiplier, compact operator, Hardy space multiplier. 
Consider for an introductory example a Paley set $P \subseteq \mathbb{N}$, i.e., a finite union of lacunary sets, or what is the same, a subset of natural numbers such that $\sup _{m}|P \cap[m, 2 m]|<\infty$. View $\ell^{\infty}(P)$ as a closed subspace of $\ell^{\infty}=\ell^{\infty}(\mathbb{N})$ simply by assigning 0 to every index $n \in \mathbb{N} \backslash P$. By Bożejko's theorem [Bo] the norm of $\Gamma_{b}$ as a Littlewood function, with $b \in \ell^{\infty}$, is equivalent to the quantity $\sup _{m}\left\{\sum_{k=m}^{2 m}|b(m)|^{2}\right\}^{1 / 2}$. The following is then immediate.

Proposition. The Hankel-Schur multiplier $\Gamma_{b}$ with $b \in \ell^{\infty}(P)$ is compact if and only if $b \in c_{0}(P)$.

Writing $b=b_{1} b_{2}$ coordinate-wise with $b_{j} \in c_{0}(P)$, a result of Rudin $[\mathrm{R}$ Thm. 2.1] supplies some $f \in L^{1}(\mathbb{T})$ such that $\left.\hat{f}\right|_{P}=\left.b_{2}\right|_{P}$, since $P$ is a Sidon set. Thus $\Gamma_{b}$ with $b \in \ell^{\infty}(P)$ is a compact Schur multiplier if and only if it factors as $\Gamma_{b}=\Gamma_{b_{1}} * \Gamma_{\widehat{f}}$. This is in fact a general phenomenon, as the main result displays.

Let the matrix $F_{N}$ have elements $f_{j k}=\max \left\{0,(N+1)^{-1}(N+1-j-k)\right\}$. Then $F_{N}=\Gamma_{\widehat{k_{N}}}$ is a Hankel matrix and corresponds to the positive Fejér kernel $k_{N}$. It follows that $\left\|F_{N}\right\|_{m}=\left\|k_{N}\right\|_{L^{1}}=1$, all $N \geq 0$.

Theorem. Let the Schur multiplier A be given. Then the following statements are equivalent.

a. $A: V_{2} \rightarrow V_{2}$ is compact.

b. For some subsequence $N(j) \rightarrow \infty$ the convergence $\left\|A-F_{N(j)} * A\right\|_{m} \rightarrow 0$ is obtained.

c. $\left\|A-F_{N} * A\right\|_{m} \rightarrow 0$ as $N \rightarrow \infty$.

d. $A=\Gamma_{\hat{f}} * B$, where $B: V_{2} \rightarrow V_{2}$ is a (compact) Schur multiplier and $\hat{f}$ is the Fourier sequence given by some integrable $f \in L^{1}(\mathbb{T})$.

Proof. Keep in mind that $\left\{F_{N}\right\}_{N=0}^{\infty}$ is a bounded set in $V_{2}$. Since statement b. expresses $A$ as a limit of finite rank multipliers, clearly a. and b. are equivalent. On the other hand, any accumulation point of any sequence $\left\{F_{N(j)} * A\right\}_{1}^{\infty}$ is necessarily represented by the same matrix as $A$ is, so standard functional analysis equates statements b. and c. Presupposing statement d., one has

$$
\left\|A-F_{N} * A\right\|_{m}=\left\|B *\left(\Gamma_{\widehat{f}}-F_{N} * \Gamma_{\widehat{f}}\right)\right\|_{m} \leq\|B\|_{m}\left\|f-\sigma_{N} f\right\|_{M(\mathbb{T})} \rightarrow 0
$$

as $N \rightarrow \infty$. Here $\sigma_{N} f$ is the $N$-th Fejér sum of $f \in L^{1}(\mathbb{T})$. Hence $A$ is compact by the previous argument.

Finally, the action $L^{1}(\mathbb{T}) \times V_{2} \rightarrow V_{2},(f, A) \mapsto \Gamma_{\widehat{f}} * A$ introduces on $V_{2}$ a structure of an $L^{1}(\mathbb{T})$-module, fully compatible with the multiplier norm topology. Likewise the closed subalgebra of compact multipliers becomes a module under this action:

$$
\left\|\Gamma_{\widehat{f}} * A\right\|_{m} \leq\left\|\Gamma_{\widehat{f}}\right\|_{m}\|A\|_{m} \leq\|f\|_{L^{1}}\|A\|_{m}
$$

Since the range of this action is dense in the space of compact multipliers, the Module Factorization Theorem of Cohen-Hewitt (cf. [HR]) says exactly that statement d. holds provided $A$ indeed should be compact. The proof is complete.

Corollary. Any compact Hankel-Schur multiplier is approximable by finitely supported Hankel-Schur multipliers.

Proof. Any $F_{N} * A$ is Hankel-shaped if $A$ is so! 
Additionally, the same proof generalizes mutatis mutandis to handle the multiplier spaces $M\left(H^{1}\right)$ and $M\left(L^{1}\right) \simeq M(\mathbb{T})$. The first statement below is of course known to everyone.

Proposition. A multiplier $a \in M\left(L^{1}\right)$ is a compact mapping $L^{1} \rightarrow L^{1}$, as well as $M\left(L^{1}\right) \rightarrow M\left(L^{1}\right)$, if and only if $a=\hat{f}$ for some $f \in L^{1}(\mathbb{T})$. Correspondingly, a multiplier $a \in M\left(H^{1}\right)$ is a compact mapping $M\left(H^{1}\right) \rightarrow M\left(H^{1}\right)$ if and only if there are $b \in M\left(H^{1}\right)$ and $f \in L^{1}(\mathbb{T})$ such that $a(n)=b(n) \hat{f}(n)$, all $n \geq 0$.

Observe that the above discrepancy with integrable factors for the algebras $V_{2}$ and $M\left(H^{1}\right)$, but identification with Fourier sequences for the algebra $M\left(L^{1}\right)$, is necessary in order to accommodate the compact, Paley set-based multipliers. For another source of examples - somewhat surprising - a classification of the author comes in handy:

Lemma $([\mathrm{A}])$. The Riesz product measure $\mu=\prod_{k=1}^{\infty}\left(1+a_{k} e^{i n_{k} \theta}+\overline{a_{k}} e^{-i n_{k} \theta}\right)$, where $\left\{n_{k}\right\}$ is lacunary, defines a multiplier $\left.\hat{\mu}\right|_{\mathbb{N}} \in M\left(H^{1}, H^{2}\right)$ if and only if

$$
\sup _{k}\left|a_{k}\right|^{2} \prod_{j=1}^{k-1}\left(1+2\left|a_{k}\right|^{2}\right)<\infty \text {. }
$$

Lemma ([A] Examples 8 and 9]). There exist singular Riesz products that generate multipliers in $M\left(H^{1}, H^{2}\right)$.

By Bożejko's theorem, membership in $M\left(H^{1}, H^{2}\right)$ above can freely be replaced by being a Littlewood-Hankel function in $T_{2}^{H}$, a certain subspace of HankelSchur multipliers. It is fairly straightforward to verify that every multiplier in $M\left(H^{1}, H^{2}\right)$ is weakly compact when seen as acting $H^{1} \rightarrow H^{1}$; in particular, every Paley set makes the members of $\ell^{\infty}(P) \subset M\left(H^{1}\right)$ weakly compact and those in $c_{0}(P) \subset M\left(H^{1}\right)$ compact multipliers. In contrast, $\ell^{\infty}(P) \backslash c_{0}(P) \subset M^{H}$ generates non-weakly compact Hankel-Schur multipliers. This can be verified by studying permutation operators on $\ell^{2}$.

Now let $\mu$ satisfy the last lemma; by singularity $\sum\left|a_{k}\right|^{2}=\infty$. Trivially there exists a decreasing null-sequence $\left.\left.\left\{b_{k}\right\} \subset\right] 0,1\right]$ such that $\sum\left|a_{k} b_{k}\right|^{2}=\infty$ still holds. Let the Riesz product $\nu$ correspond to $\left\{a_{k} b_{k}\right\}_{1}^{\infty}$. By construction $\nu$ is singular and

$$
\left|a_{k} b_{k}\right|^{2} \prod_{j=1}^{k-1}\left(1+2\left|a_{k} b_{k}\right|^{2}\right) \leq b_{k}^{2} \cdot \mathcal{O}(1)=o(1)
$$

Therefore the technique of [A, Proposition 4] shows that $\left.\hat{\nu}\right|_{\mathbb{N}}$ produces a compact Hankel-Schur multiplier $\Gamma_{\widehat{\nu} \mid \mathbb{N}}$, and in fact the truncation $\hat{\nu}_{M}=\hat{\nu} \chi_{[0, M]}$ has the property

$$
\left\|\Gamma_{\widehat{\nu}}-\Gamma_{\widehat{\nu}_{M}}\right\|_{m} \leq C \sup _{m \geq M+1}\left[\sum_{k=m}^{2 m}|\hat{\nu}(k)|^{2}\right]^{1 / 2} \rightarrow 0 \quad \text { as } \quad M \rightarrow \infty .
$$

Proposition. There exist compact Hankel-Schur multipliers that are generated by Fourier-Stieltjes sequences of singular measures.

Observe that in the restricted Fourier-Stieltjes algebra $B(\mathbb{N})$ the norm inequality $\left\|\hat{\nu}-\hat{\nu}_{M}\right\|_{B(\mathbb{N})} \geq 1$ holds for all $M \geq 0$, since $\nu$ is singular and of total variation 1 . Therefore the compactness of $\Gamma_{\widehat{\nu}}$ is a phenomenon in the multiplier algebra $V_{2}$ and is not due to any conditions inside $B(\mathbb{N})$ or $M(\mathbb{T})$. 
Applying the theorem above, the stated compactness as a multiplier produces some compact $b \in M^{H}$ and $f \in L^{1}(\mathbb{T})$ such that $\hat{\nu}(n)=b(n) \hat{f}(n)$, all $n \geq 0$. However, the singularity of $\nu$ as a measure prevents the factor $b$ from being a member of $B(\mathbb{N})$, so $b$ cannot possibly be the restriction to $\mathbb{N}$ of a Fourier-Stieltjes transform. One should note that the support of $b$ is not contained in any Paley set, since this is true of $\left.\hat{\nu}\right|_{\mathbb{N}}$.

Corollary. There exist singular measures $\nu \in M(\mathbb{T})$ that are divisible by integrable functions in the sense that $\hat{\nu}(n)=b(n) \hat{f}(n)$, all $n \geq 0$, for a suitable function $f \in L^{1}(\mathbb{T})$ and a sequence $b$ generating a (compact) Schur multiplier $\Gamma_{b}$.

The following material is intended to display the implications of the Paley spectrum upon Hankel-Schur multipliers. Recall first an important result of Klemes:

Theorem $([\overline{\mathrm{K}}])$. Any idempotent multiplier of $H^{1}$ is the characteristic function of a set arising as a finite boolean combination of i) arithmetic progressions, ii) finite sets, and iii) lacunary sets.

Thus the same thing holds for the diagonals of idempotent Hankel-Schur multipliers. Invoking Bożejko's criterion, the largest sub- $C^{*}$-algebras of $M^{H}$ may be identified using a straightforward argument:

Corollary. Any set $E \subseteq \mathbb{N}$ that allows the natural embedding $c_{0}(E) \rightarrow M^{H}$ or $c_{0}(E) \rightarrow M\left(H^{1}\right)$ to be an isomorphism onto its range, is necessarily a Paley set.

Therefore $M^{H}$ contains sub- $C^{*}$-algebras $\ell^{\infty}(P)$ and $c_{0}(P)$, on which the supremum and multiplier norms are equivalent, if and only if $P$ is a Paley set; clearly $c_{0}(P)$ consists entirely of compact Hankel-Schur multipliers.

To understand why the Fourier spectrum of the previous example with measure $\nu$ turned out by necessity to be thicker than Paley sets, some classical results from Fourier analysis come in handy. Recall that a set $E \subset \mathbb{Z}$ is a $\Lambda(2)$-set if the norm on $L_{E}^{2}(\mathbb{T})$, functions with spectrum in $E$, is equivalent to the $L^{1}$-norm.

Lemma. Let $E \subset \mathbb{N}$ be a $\Lambda(2)$-set. Then there is a positive constant $k_{E}$ such that every $a \in \ell^{\infty}$ with support in $E$ satisfies

$$
k_{E}\|a\|_{\ell^{2}} \leq\|a\|_{B(\mathbb{N})} \leq\|a\|_{\ell^{2}} .
$$

Proof. The upper inequality is immediate since $f \sim \sum_{n \in E} a_{n} e^{i n \theta}$ has

$$
\|a\|_{B(\mathbb{N})} \leq\|f\|_{L^{1}} \leq\|f\|_{L^{2}}=\|a\|_{\ell^{2}} .
$$

Let on the other hand $a \in \ell^{\infty}$ be supported in $E$ and such that $\|a\|_{B(\mathbb{N})}$ is finite. Invoking the weak-*-topology on $M(\mathbb{T})$ there is some $\nu \in M(\mathbb{T})$ with $\left.\hat{\nu}\right|_{\mathbb{N}}=a$ and $\|\nu\|_{M(\mathbb{T})}=\|a\|_{B(\mathbb{N})}$. Now $E$ is also a $\Lambda(1)$-set, since it is a $\Lambda(2)$-set, so by $[\mathrm{R}$ Thm. 5.7], the Fourier spectrum in $E \cup \mathbb{Z}_{-}$forces $\nu$ to be absolutely continuous.

Next, based on the $\Lambda(2)$-property, a theorem of Fournier $[\mathrm{F}]$ produces a positive number $k_{E}$ such that any integrable function with Fourier spectrum in $E \cup \mathbb{Z}_{-}$has $k_{E}\|\hat{f}\|_{\ell^{2}(E)} \leq\|f\|_{L^{1}}$. It follows that

$$
k_{E}\|a\|_{\ell^{2}}=k_{E}\|\hat{\nu}\|_{\ell^{2}(E)} \leq\|\nu\|_{M(\mathbb{T})}=\|a\|_{B(\mathbb{N})},
$$

which is the claimed lower inequality.

Since every Paley set is a Sidon set and thus also a $\Lambda(2)$-set, the next results are immediate. 
Corollary. Let $P \subset \mathbb{N}$ be a Paley set. Then every $a \in \ell^{\infty}(P) \subset \ell^{\infty}(\mathbb{N})$ is the diagonal of a Littlewood function and generates as such (Bożejko's criterion) a Hankel-Schur multiplier with diagonal a, but alas this multiplier corresponds to a measure if and only if $a \in \ell^{2}$.

Corollary. For a Paley set $P$, every $a \in c_{0}(P) \backslash \ell^{2}$ is the diagonal of a compact Hankel-Schur multiplier that is not generated by a measure: $\left\|\Gamma_{a}\right\|_{m} \leq C_{P}\|a\|_{\infty}$, but $\|a\|_{B(\mathbb{N})}=\infty$. In contrast, every $c \in \ell^{2}(\mathbb{N})$ corresponds via the diagonal to a compact Hankel-Schur multiplier generated by an absolutely continuous measure.

\section{REFERENCES}

[A] M.E. Andersson, Two special applications of Riesz products, Fourier analysis and related topics, vol. 56, Banach Center Publications, Warszawa, 2002, pp. 13-29. MR1971560 (2004b:42019)

[Be] G. Bennett, Schur multipliers, Duke Math. J. 44 (1977), 603-639. MF0493490 (58:12490)

[Bo] M. Bożejko, Littlewood functions, Hankel multipliers and power bounded operators on a Hilbert space, Colloq. Math. 51 (1987), 35-42. MR0891273 (88i:47011)

[F] J.J.F. Fournier, Extensions of a Fourier multiplier theorem of Paley, Pacific J. Math. 30 (1969), 415-431. MR0257651 (41:2301)

[H] M. Hladnik, Compact Schur multipliers, Proc. of Amer. Math. Soc. 128 (9) (2000), 25852591. MR 1766604 (2002a:46081)

[HR] E. Hewitt and K. Ross, Abstract harmonic analysis II, Springer Verlag, Berlin-HeidelbergNew York, 1970. MR.0262773 (41:7378)

[K] I. Klemes, Idempotent multipliers of $H^{1}(\mathbb{T})$, Canad. J. Math. 39 (5) (1987), 1223-1234. MR 0918595 (88k:42007)

[R] W. Rudin, Trigonometric series with gaps, J. Math. Mech. 9 (2) (1960), 203-227. MR.0116177(22:6972)

Bellmansgatan 118, SE-754 26 Uppsala, Sweden

E-mail address: mats@math.uu.se 